\title{
Erratum: Critical properties of a two-dimensional Ising magnet with quasiperiodic interactions [Phys. Rev. E 93, 042111 (2016)]
}

\author{
G. A. Alves, M. S. Vasconcelos, and T. F. A. Alves
}

(Received 1 December 2017; published 1 February 2018)

DOI: 10.1103/PhysRevE.97.029901

We have revised our results, we found some errors concerning the real order parameter for this system, and we have to change the main conclusions of the original paper. The first conclusion that will change is that this system does not have a spin glass phase and does not change its universality class. Instead, this system is a ferrite, and the proper critical behavior is in the Ising universality class with logarithmic corrections.

In the original article and in the first Erratum [1] we have determined the Edwards-Anderson (EA) order parameter $q_{\mathrm{EA}}$, the susceptibility $\chi$, the specific heat $c$, and the Binder cumulant $g_{L}$ by using the replica exchange Monte Carlo technique (also known as parallel tempering) [2-5], which is suited to approach the problem of determining the steady state of systems with complex energy landscapes composed of many local minima and to find the ground state of systems with nonperiodic interactions.

But, by analyzing the same system with the Octonacci sequence [6], to be published elsewhere, we calculated the dynamical correlation to see if the system exhibits slow dynamics, i.e., aging. But the system exhibits aging for ferromagnetic systems [7], so, unlike the way in which we concluded in our original article, the system is ferromagnetic, and its ground state is an aperiodic ferrite. The correct order parameter is the staggered magnetization. We were motivated by the change in the specific heat critical behavior and, apparently, we equivocally concluded that the critical exponents for $q_{\mathrm{EA}}$ matched the critical exponent for specific heat. However, as we show, the critical behavior of the specific heat is changed by logarithmic corrections.

By using the replica exchange Monte Carlo technique, we obtained the staggered magnetization order parameter $\langle q\rangle$, the associated susceptibility $\chi$, the specific heat $c$, and the Binder cumulant $g$,

$$
\begin{gathered}
q=\frac{1}{N^{*}} \sum_{i}^{N} S_{i}^{0} S_{i}, \\
\chi=N\left(\left\langle q^{2}\right\rangle-\langle q\rangle^{2}\right) / T, \\
g=1-\frac{\left\langle q^{4}\right\rangle}{3\left\langle q^{2}\right\rangle^{2}},
\end{gathered}
$$

where $\langle\cdots\rangle$ stands for a thermal average over sufficiently many independent steady state system configurations, $S_{i, j}^{0}$ is the ground state of the system, and $L$ and $T$ are the lattice size and the absolute temperature, respectively. Equations (1)-(3) replace (2)-(5) of our original article. We use the same values of the lattice size $L: 34,55,89,144$, and 233, which are Fibonacci numbers $F_{n}$, given by the recursion rule,

$$
F_{n}=F_{n-1}+F_{n-2},
$$

where $F_{0}=1$ and $F_{1}=1$.

To determine the proper critical behavior, we have used the following finite size scaling (FSS) relations [8] with logarithmic corrections [9-12],

$$
\begin{gathered}
q \propto L^{\beta / v}(\ln L)^{\hat{\beta}+\beta \hat{\lambda}} f_{q}(\vartheta), \\
\chi \propto L^{-\gamma / v}(\ln L)^{\hat{\gamma}-\gamma \hat{\lambda}} f_{\chi}(\vartheta), \\
c \propto(\ln L)^{-\hat{\alpha}} f_{c}(\vartheta),
\end{gathered}
$$

where $\beta=1 / 8, \gamma=7 / 4, \alpha=0$ (logarithmic divergence), and $v=1$ are the critical exponents [the Ising two-dimensional (2D) ones]. $\hat{\alpha}, \hat{\beta}, \hat{\gamma}$, and $\hat{\lambda}$ are the logarithmic correction exponents. Equations (5)-(7) replace (6)-(8) of our original article. $f_{i}(\vartheta)$ 's are the FSS functions with a logarithmic

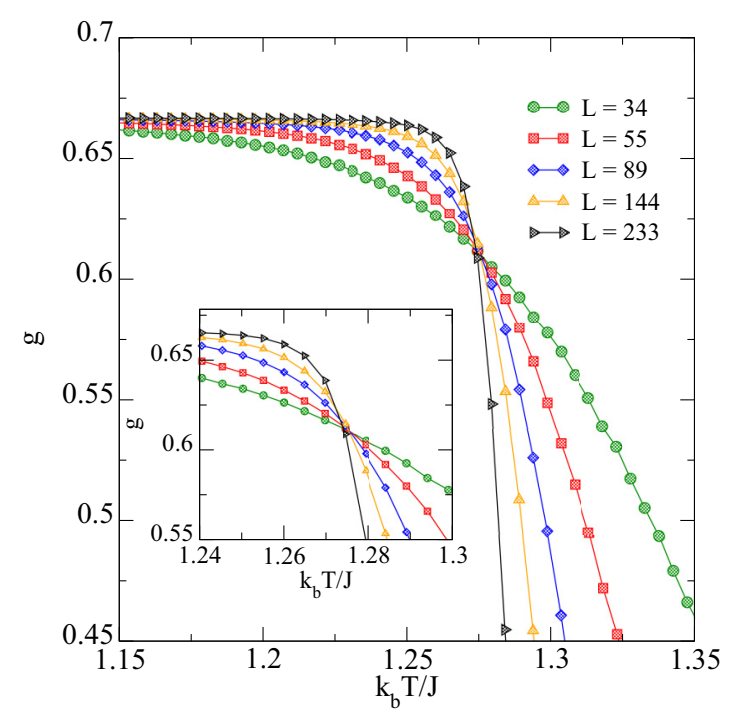

FIG. 1. Binder cumulant $g$ versus temperature $T$ for different lattice sizes $L$. The values of $L$ obey the Fibonacci sequence. We estimated the critical temperature of $T_{c} \approx 1.274$ by averaging the numerical values of the temperatures where the curves intersect each other. 


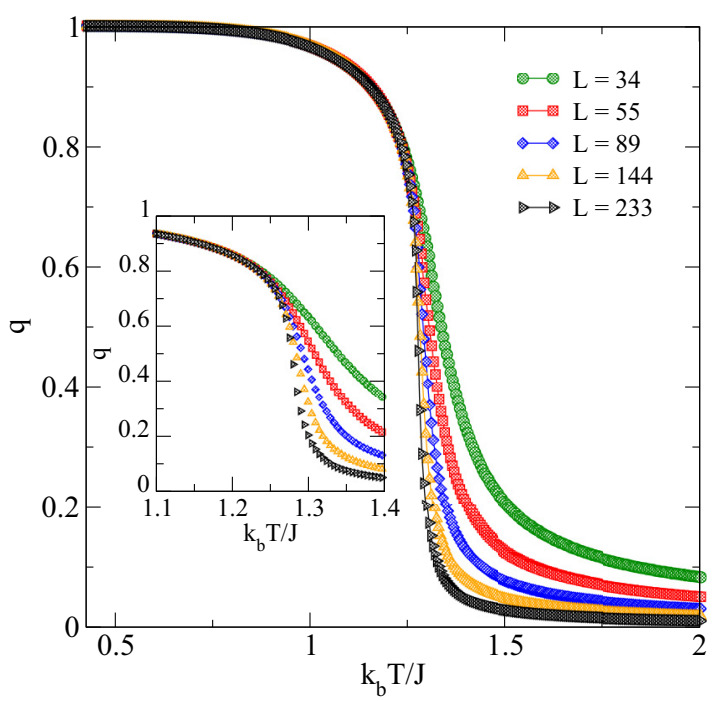

FIG. 2. The order parameter $q$ as a function of temperature $T$ for different lattice sizes $L$. The values of $L$ obey the Fibonacci sequence. The inset: The curves suggest a second-order phase transition.

corrected scaling variable,

$$
\vartheta=L^{1 / v}\left(T-T_{c}\right)|\ln | T-\left.T_{c}\right|^{-\hat{\lambda}} .
$$

Equation (8) replaces (9) of our original article. The correction exponents $\hat{\alpha}, \hat{\beta}, \hat{\gamma}$, and $\hat{\lambda}$ obey the following scaling relations [12]:

$$
\begin{gathered}
\hat{\alpha}=1-d \nu \hat{\lambda}, \\
2 \hat{\beta}-\hat{\gamma}=-d \nu \hat{\lambda},
\end{gathered}
$$

where $d=2$ is the dimensionality of the system. The scaling relation (9) is valid only for $\alpha=0$ (logarithmic divergences) in the general case of $\hat{\alpha}=-d \nu \hat{\lambda}$. For $\alpha=0$ and $\hat{\alpha}=0$, we

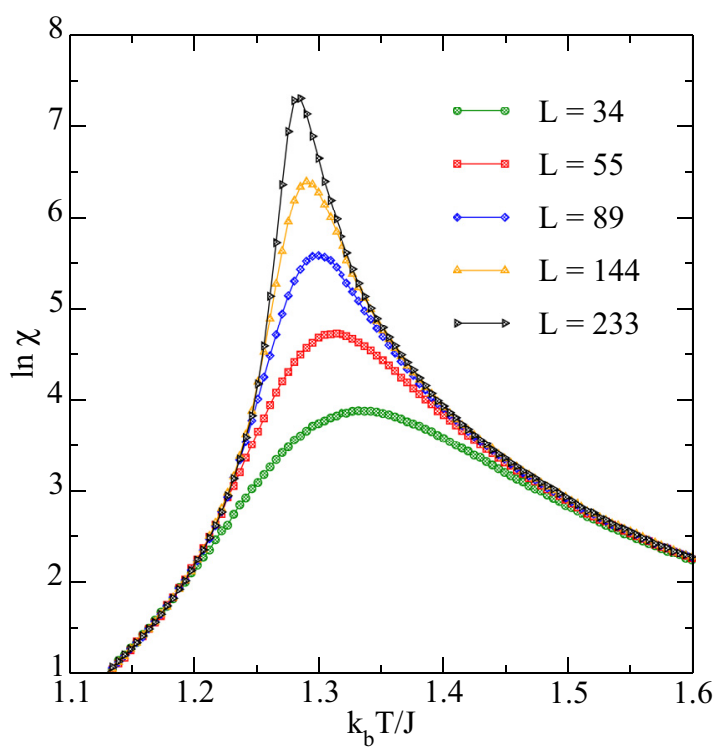

FIG. 3. The susceptibility $\chi$ as a function of temperature $T$ for different lattice sizes $L$. The values of $L$ obey the Fibonacci sequence. The inset: The susceptibility diverges at $T_{c}$ in the large lattice size limit.

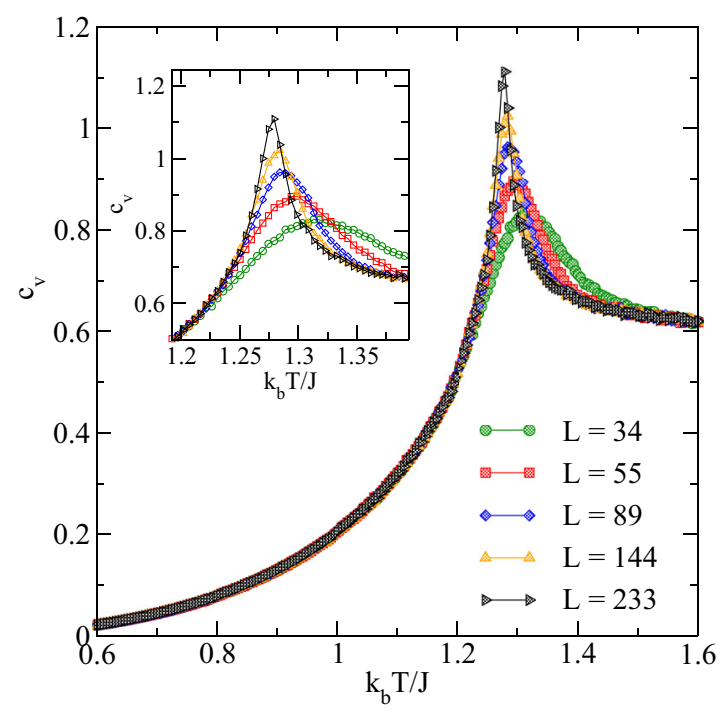

FIG. 4. Specific heat $c_{v}$ as a function of temperature $T$ for different lattice sizes $L$. When increasing the lattice size, we observe a crescent maximum, suggesting a logarithm divergence at the critical temperature of $T_{c} \approx 1.274$.

have the double logarithmic divergence $(\ln \ln L)$ of the specific heat as seen for the 2D diluted Ising model [12]. Because of the changing of the proper order parameter from $q_{\mathrm{EA}}$ to the staggered magnetization $q$ and the changing of the scaling properties to take into account the logarithmic corrections, Figs. 2, 5, 8, and 9 of our original article should not be considered.

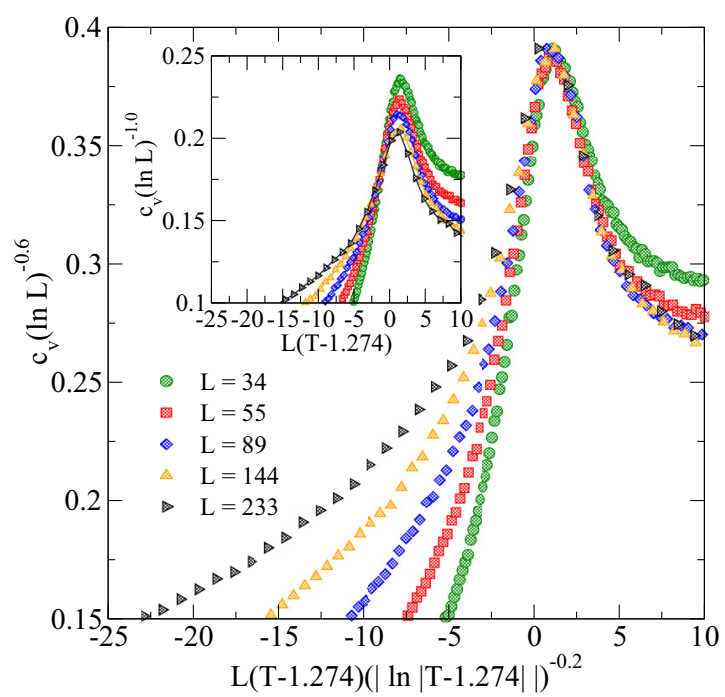

FIG. 5. Data collapse of the specific heat $c$, rescaled by $(\ln L)^{-\hat{\alpha}}$ versus the scaling parameter $L^{1 / v}\left(T-T_{c}\right)|\ln | T-T_{c} \|^{-\hat{\lambda}}$ for different lattice sizes $L$. The inset: Specific heat $c$, rescaled by $1 / \ln L$ versus the scaling parameter $L^{1 / v}\left(T-T_{c}\right)$ for different lattice sizes $L$. We can see that the FSS relation without logarithmic corrections does not collapse our numerical data. The best collapse is performed by using the values for the logarithmic correction exponents: $\hat{\alpha}=3 / 5$ and $\hat{\lambda}=1 / 5$. The model is in the Ising universality class with logarithmic corrections. 

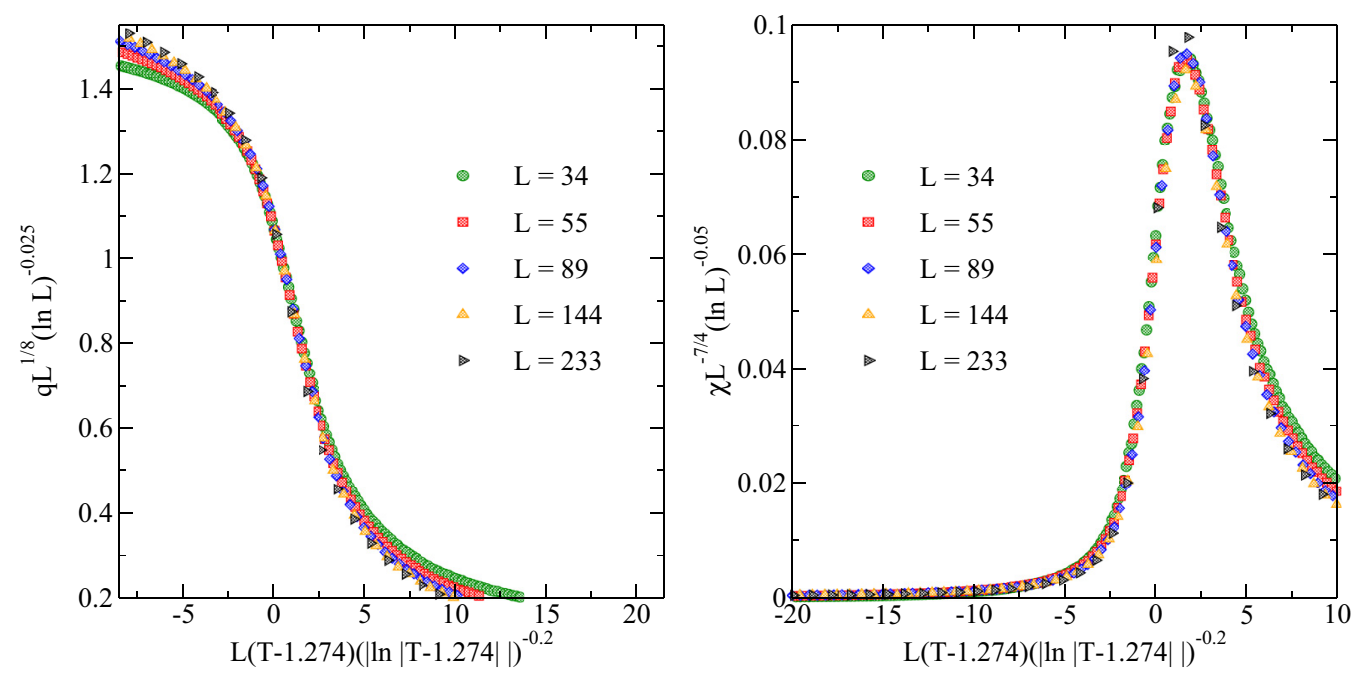

FIG. 6. Data collapse of the order parameter $q$ and the susceptibility $\chi$, rescaled by $L^{\beta / v}(\ln L)^{\hat{\beta}+\beta \hat{\lambda}}$ versus the scaling parameters $L^{1 / \nu}(T-$ $\left.T_{c}\right)|\ln | T-\left.T_{c}\right|^{-\hat{\lambda}}$ and $L^{-\gamma / v}(\ln L)^{\hat{\gamma}-\gamma \hat{\lambda}}$ versus the scaling parameter $L^{1 / v}\left(T-T_{c}\right)|\ln | T-\left.T_{c}\right|^{-\hat{\lambda}}$, respectively, for different lattice sizes $L$. The best collapse is performed by using the values for the logarithmic correction exponents: $\hat{\beta}=-1 / 20, \hat{\gamma}=3 / 10$, and $\hat{\lambda}=1 / 5$. Therefore, the model is in the Ising universality class with logarithmic corrections.

We have used $1 \times 10^{5}$ Monte Carlo Markov (MCM) steps to make the $N_{t}=600$ system replicas (each system replica has a different temperature) reach the equilibrium state, and the independent steady state system configurations are estimated in the next $1 \times 10^{6}$ MCM steps with ten MCM steps between one system state and another one to avoid self-correlation effects. Every MCM step is composed of two parts, a sweep and a swap. One sweep is accomplished when all $N$ spins were investigated if they flip or not, and one swap is accomplished if all the $N_{t}$ replicas are investigated if they exchange or not their temperatures (swap part). We carried out $10^{5}$ independent steady state configurations to calculate the needed thermodynamic averages.

We have reestimated the critical temperature by using the Binder cumulant given by Eq. (3) for the staggered magnetization in order to obtain the critical temperature. We show the Binder cumulant in Fig. 1 which replaces Fig. 3 of our original article. The critical temperature $T_{c}$ is estimated at the point where the curves for different size lattices intercept each other as shown in the inset. We obtained $T_{c} \approx 1.274$ by using the Binder cumulant for the staggered magnetization.

We display the order parameter $q$ versus temperature $T$ in Fig. 2 which replaces Fig. 4 of the our first Erratum [1]. The inset shows the original data for staggered magnetization $q$, and the main figure shows data collapse by using the FSS relation written in Eq. (5) in Fig. 6.
Continuing the analysis of the critical behavior, we obtained the susceptibility $\chi$ as a function of temperature $T$, Fig. 3 which replaces Fig. 7 of our original article. In the large lattice size limit, the susceptibility diverges at $T_{c} \approx 1.274$. Also, we show the data collapse of the susceptibilities for different lattice sizes according to the FSS relation given in Eq. (6). All maxima are well fitted by using the FSS relation with logarithmic corrections and the Ising critical exponents as shown in Fig. 6. This figure replaces Fig. 12 of the our first Erratum [1].

Finally, we show the specific heat $c$ as a function of temperature $T$, Fig. 4 which replaces Fig. 10 of our original article. We have estimated the $\hat{\alpha}$ exponent by collapsing the specific heat $c$ for different lattice sizes following the scaling relation presented in Eq. (7) as we show in Fig. 5. We have noted that the maxima of the specific heat diverges as a power of $\ln L$ as shown in Fig. 5, unlike the pure model in which the maxima scales as $\ln L$ as shown in the inset of Fig. 5, which substitutes for Fig. 11 of our original article. When using the exponent of the pure model without logarithmic corrections, our numerical data are not collapsed. Our best estimate for the $\hat{\alpha}$ exponent ratio is $\hat{\alpha}=3 / 5$, which obeys the scaling relations for the logarithmic correction exponents given in Eq. (7). Choosing the order parameter, the susceptibility and the specific heat logarithmic correction exponents preserve the scaling relations Eqs. (9) and (10). We show the data collapsed for the staggered magnetization and its susceptibility in Fig. 6, which substitutes for Fig. 12 of our original article.
[1] G. A. Alves, M. S. Vasconcelos, and T. F. A. Alves, Phys. Rev. E 94, 019904(E) (2016).

[2] D. J. Earl and M. W. Deem, Phys. Chem. Chem. Phys. 7, 3910 (2005).

[3] R. H. Swendsen and J.-S. Wang, Phys. Rev. Lett. 57, 2607 (1986).
[4] K. Hukushima and K. Nemoto, J. Phys. Soc. Jpn. 65, 1604 (1996).

[5] C. J. Geyer, in Computing Science and Statistics Proceedings of the 23rd Symposium on the Interface (American Statistical Association, New York, 1991). 
[6] G. A. Alves, M. S. Vasconcelos, and T. F. A. Alves, J. Stat. Mech. (2017) 123302.

[7] H. Rieger, B. Steckemetz, and M. Schreckenberg, Europhys. Lett. 27, 485 (1994).

[8] F. W. S. Lima, Physica A 391, 1753 (2012).

[9] R. Kenna, D. A. Johnston, and W. Janke, Phys. Rev. Lett. 96, 115701 (2006).
[10] R. Kenna, D. A. Johnston, and W. Janke, Phys. Rev. Lett. 97, 155702 (2006).

[11] V. Palchykov, C. von Ferber, R. Folk, Y. Holovatch, and R. Kenna, Phys. Rev. E 82, 011145 (2010).

[12] R. Kenna, Universal Scaling Relations for LogarithmicCorrection Exponents (World Scientific, London, 2012), Vol. 3, Chap. 1, p. 1. 